\title{
The influenza virus hemagglutinin: a model protein in the study of membrane fusion
}

\author{
João Ramalho-Santos ${ }^{\mathrm{a}, \mathrm{b}}$, Maria C. Pedroso de Lima ${ }^{\mathrm{a}, \mathrm{c}, *}$ \\ a Center for Neuroscience of Coimbra, University of Coimbra, Coimbra, Portugal \\ ${ }^{\mathrm{b}}$ Department of Zoology, University of Coimbra, Coimbra, Portugal \\ ${ }^{c}$ Department of Biochemistry, University of Coimbra, Coimbra, Portugal
}

Received 29 September 1997; revised 6 January 1998; accepted 13 February 1998

Keywords: Influenza virus; Hemagglutinin; Membrane fusion

\section{Contents}

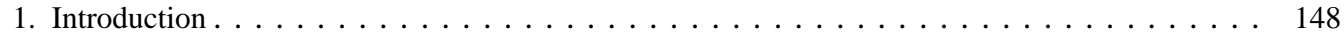

2. A working model for influenza hemagglutinin fusion activity . . . . . . . . . . . . . 150

2.1. First contact: role of target membrane viral receptors in membrane merging . . . . . . . 150

2.2. Turning it on: low $\mathrm{pH}$ conformational changes in the HA structure . . . . . . . . . . . . 150

2.3. Disturbing influences: insertion of the viral fusion peptide into the target membrane

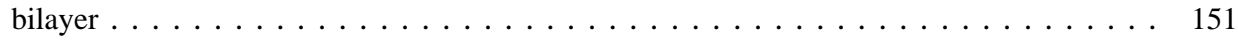

2.4. Key cooperations: participation of several HA trimers at the fusion site . . . . . . . . 151

2.5. A question of bulk: overcoming physical barriers to final contact between membranes . . . 152

2.6. Involvement of proteins and lipids: early intermediates in the coalescence of aqueous

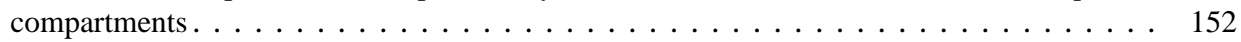

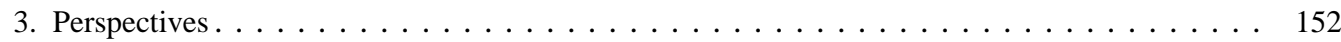

Acknowledgements . . . . . . . . . . . . . . . . . . . . . . . . 153

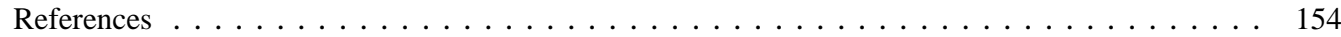

\section{Introduction}

Membrane fusion is involved in a wide variety of biological processes, from sperm-oocyte fusion, to myoblast fusion during muscle development, to a large number of intracellular membrane trafficking

\footnotetext{
${ }^{*}$ Corresponding author. Department of Biochemistry, Apartado 3126, University of Coimbra, 3000 Coimbra, Portugal. Fax: +351-39-4192607; E-mail: mdelima@cygnus.ci.uc.pt
}

events that participate in protein synthesis and targeting, as well as in endo and exocytosis. However, the best characterized fusion process relates to cell infection by lipid-enveloped viruses, namely to the merging of the viral envelope with a target cell membrane, resulting in viral nucleocapsid delivery into the cytoplasm. This is due to the simplicity of the system, where generally only one or two envelope proteins (present in large quantities) mediate bilayer mixing. Of all the lipid enveloped viruses that have been 
studied, influenza virus has been, by far, the major source of information, and its sole fusogenic protein, designated hemagglutinin (HA), has therefore become a paradigmatic model in this field of research.

Influenza virus enters target cells by receptormediated endocytosis, and is delivered to an intracellular acidic organelle, the endosome. The low $\mathrm{pH}$ of this compartment triggers a massive conformational change in the HA, rendering it fusion-active, and thus promoting merging between the viral envelope and the endosomal membrane [1-3]. The entry of influenza virus into target cells has therefore a small time window, taking place only after arrival of infectious particles in the endosome, and requiring completion before the virus comes into contact with lysosomal hydrolases. This may explain the fact that influenza virus, as other low $\mathrm{pH}$-dependent viruses, normally fuses very quickly and effectively, when compared with viruses that enter target cells by direct fusion between the envelope and the cell plasma membrane at neutral $\mathrm{pH}$, such as Sendai virus [4-6].

Influenza virus HA is a homotrimeric glycoprotein synthesized in infected cells as an inactive precursor (designated HA0), and only becomes fusion-competent when cleaved into two subunits, HA1 and HA2, joined by disulfide bonds [7]. At neutral $\mathrm{pH}$, HA trimers form spikes that project $\pm 130 \AA$ from the viral envelope (Fig. 1A, $\mathrm{A}^{\prime}$ ), each monomer containing a globular head region (HA1), responsible for viral binding to target membrane sialic acid (the biological receptor for the virus), and an envelope- anchored stem region (HA2). As mentioned above, HA-mediated membrane fusion only takes place following a protein conformational change at low $\mathrm{pH}$, occurring in at least two steps (Fig. 1B, $\mathrm{B}^{\prime}$ ) [8]: exposure of a short hydrophobic peptide $( \pm 20$ a.a. $)$ localized at the $\mathrm{N}$-terminal of HA2, and that was previously buried inside the protein, and dissociation of the globular heads (i.e., each monomeric HA1 drifts apart from the other two). The hydrophobic peptide is essential for membrane merging [9], and has thus been dubbed the 'fusion peptide'. It is generally agreed that, under acidic conditions as found in the endosome, the HA conformational change takes place quickly, is irreversible, and if the protein cannot trigger fusion (due to the absence of a target membrane, or to poor positioning of the virus), its activity is also rapidly and irreversibly lost, a process known as viral inactivation $[1,5]$. However, recent data seem to suggest that at low $\mathrm{pH}$, HA may maintain its fusogenic ability for long periods of time, and be therefore capable to mediate membrane mixing, albeit to a much lesser degree, several hours after the conformational change has been triggered [6]. The possibility that at least some aspects of the conformational change, namely related to the positioning of $\mathrm{HA}$ in relation to its putative target membrane at neutral and acidic $\mathrm{pH}$, may be reversible, has also been noted [10]. Another proposal suggests that only very few HA trimers on the envelope participate in fusion, and actually undergo physiologically relevant $\mathrm{pH}-$ dependent structure alterations [11].

Fig. 1. A model for the mechanism of influenza virus hemagglutinin (HA)-mediated membrane fusion. Left: Specific binding of influenza HA (green) occurs via interactions of the globular domains of HA (HA1) with sialic acid residues located on either glycoproteins or glycolipids of the target membrane at neutral $\mathrm{pH}$ (blue dots, A). At low $\mathrm{pH}$, the HA conformational change is triggered, resulting in exposure of the hydrophobic HA2 N-terminal peptide and its projection towards the target membrane at the tip of the newly formed long helix (red), and concomitant (partial) opening of the trimer (B). Beside the conformational change per se active HAs are drawn together, clustering at a 'fusion site' (B, C). Further separation of the HA1 'heads' and the tilted positioning of the HA at low pH would result in bringing the two bilayers closer together (C). Initial membrane merging involves a lipidic intermediate, with mixing of the external leaflets of both bilayers (hemifusion, D). Ultimately, the hemifusion stage will evolve into a single monolayer contact region that later collapses into a small fusion pore (E). The further enlargement of the pore (F) is possibly due to the action of the fusion peptide. Note that the peptide has now virtually reversed its initial low $\mathrm{pH}$ orientation. Right: In the absence of target membrane sialic acid, virus binding at neutral $\mathrm{pH}$ takes place unspecifically $\left(\mathrm{A}^{\prime}\right)$. Therefore, when fusion is triggered at acidic $\mathrm{pH}$, the HA conformational change does not necessarily result in membrane merging $\left(\mathrm{B}^{\prime}, \mathrm{C}^{\prime}\right)$. Under these conditions, although some HA trimers retain normal activity ( $\mathrm{B}^{\prime}$, left), others cannot promote insertion of the fusion peptide into the target bilayer (possibly due to inaccurate positioning relative to the target membrane, $\mathrm{B}^{\prime}$, right), and the N-terminal peptide of HA2 may therefore 'turn around' and insert into the viral envelope ( $\mathrm{C}^{\prime}$, right), a process that has been linked to viral inactivation. Under these conditions, there will be a low probability for correct clustering of HAs with the fusion peptide properly inserted in the target membrane, thus explaining the low fusion activity of influenza virus towards target membranes that lack specific viral receptors. (See text for further discussion). 

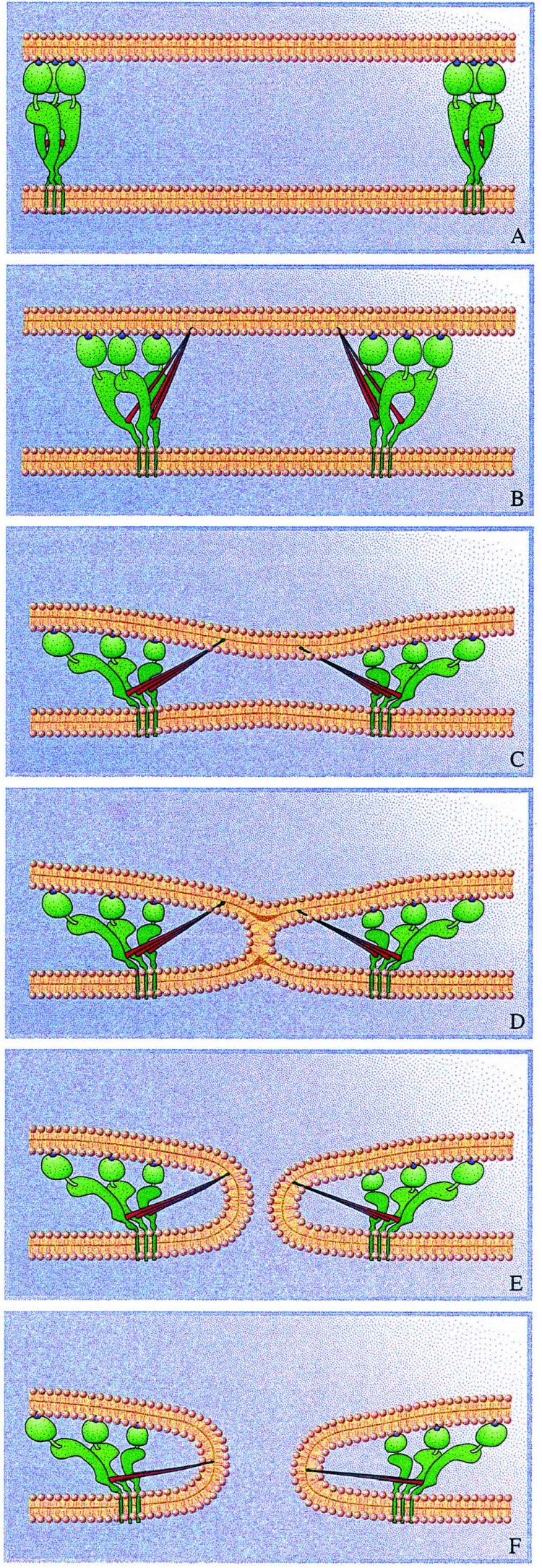
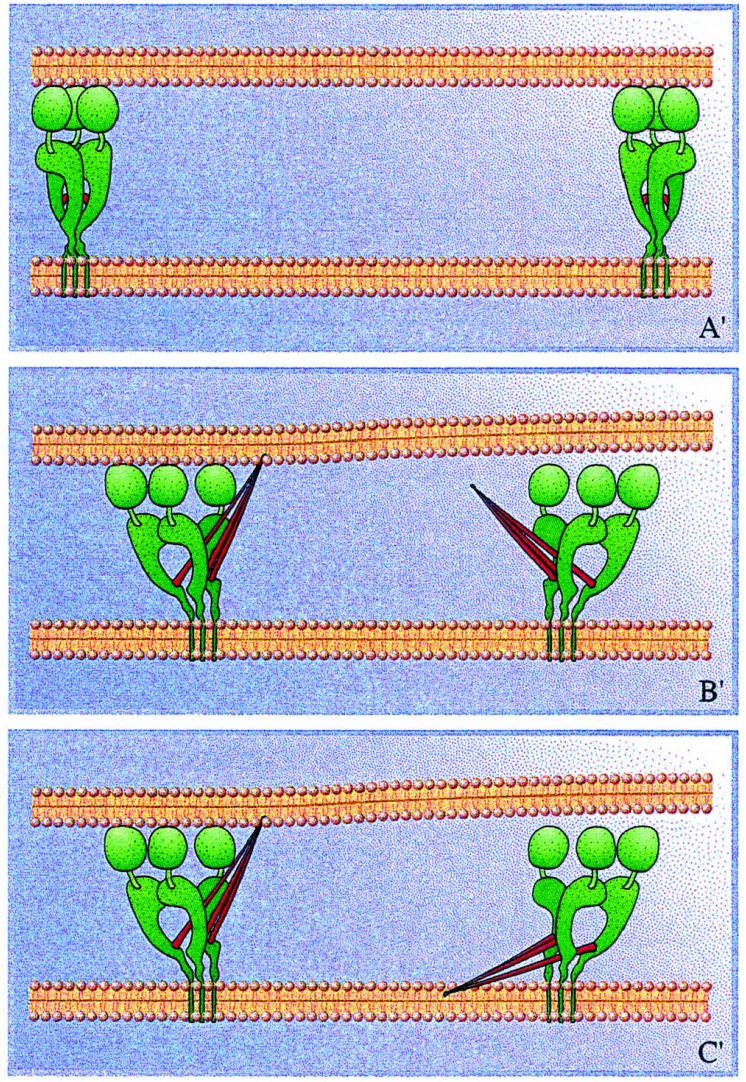
Although much information has already been obtained, complete elucidation of the molecular mechanism of membrane fusion as mediated by the influenza HA has been hindered by several constraints. One important issue is the limited structural data available, especially with respect to the fusion-active low $\mathrm{pH}$ conformation of the protein (see below). Another aspect concerns the many different systems used to probe the activity of HA, and by the concomitant difficulty in bringing together data from several experimental frameworks. These systems include either intact virions, reconstituted HA in lipid mono or bilayers, or cells expressing HA on their plasma membrane fusing with a wide variety of artificial and biological target membranes, fusion being quantified with an array of different methods.

There are limitations to virtually all of the systems mentioned. Thus, in vivo virions are subject to endocytosis, a gradual drop in $\mathrm{pH}$, following which they fuse with the inner leaflet of a target membrane (the endosomal bilayer), conditions never met in kinetic studies of viral fusion activity. In addition, many assays employing intact virions have used nonphysiological approaches (virus-target membrane prebinding in the cold at neutral $\mathrm{pH}$, viral fusion activity monitored at very low temperatures, abrupt $\mathrm{pH}$ adjustments during fusion) in an effort to slow down the process, and to rend it more amenable to analysis. Several stages in HA-mediated fusion have been characterized in this manner, although their exact physiological relevance is open to discussion. On the other hand, while the use of cells expressing HA is paramount in obtaining certain data (such as in patch clamp studies of fusion pore formation), it is obvious that they do not accurately represent the normal organization of the fusogenic protein in the intact virus, both in terms of protein density, association with other viral proteins, not to mention the existence on the plasma membrane of many other (cellular) proteins. Finally, it should also be noted that HAs from different viral strains may have slightly distinctive properties [11-14]. This being said, and although the precise nature and characteristics of a given system under study is obviously important, influenza HA will most likely mediate fusion always in the same basic manner, irrespective of experimental conditions and viral strains. Any model proposed to explain its action must, thus, be a global model, including data from all sources available, and accounting for the behavior of the fusogenic protein as a whole. Based on the recent publication of new and challenging observations, such a model is presented and discussed below (Fig. 1). The relevance of membrane fusion mediated by the HA in terms of extrapolating results obtained with this model protein to other biological membrane merging events will also be briefly evaluated.

\section{A working model for influenza hemagglutinin fusion activity}

\subsection{First contact: role of target membrane viral receptors in membrane merging}

The first step in HA-mediated membrane fusion consists of binding between the viral envelope and a target membrane at neutral $\mathrm{pH}$. Binding occurs via the HA1 globular subunits, which specifically recognize sialic acid residues on the target bilayer (Fig. 1A) [1,7], but can also take place unspecifically, i.e., via non-sialic acid containing sites (Fig. 1 $\mathrm{A}^{\prime}$ ) [15]. Specific binding is seldomly discussed in terms of the low $\mathrm{pH}$-dependent fusion activity of influenza virus. However, it has been shown that target membrane sialic acid can directly modulate HA-mediated membrane merging [15], strongly suggesting that specific viral attachment is crucial for correct HA organization at the fusion site $[15,16]$. Other suggestions imply that bound HAs do not directly mediate the fusion process, but instead help create the correct environment and membrane proximity for the bilayer merging action of non-bound trimers, possibly by allowing their clustering at the fusion site $[17,18]$ (see below).

\subsection{Turning it on: low pH conformational changes in the HA structure}

Following viral binding and endocytosis, the HA undergoes the low $\mathrm{pH}$-induced conformational change, which, as mentioned earlier, involves both exposure of the hydrophobic N-terminal fusion peptide from HA2, and dissociation of the trimer globular HA1 subunits (trimer opening; Fig. 1B, $\mathrm{B}^{\prime}$ ). The importance of these two phenomena have been widely discussed. Thus, while fusion peptide exposure was 
considered essential for membrane merging, trimer opening was dubbed irrelevant, and only involved in low-pH viral inactivation $[19,20]$. However, two lines of evidence seem to contradict this possibility. On one hand, inhibition of globular head dissociation (by linking trimer HA1 subunits via disulfide bonds) was shown to greatly impair fusion, due to a lack of fusion peptide exposure [21,22]. On the other hand, kinetic analysis strongly suggested that both low $\mathrm{pH}$-dependent fusion activity and inactivation shared a common molecular mechanism, and thus relied on similar structural changes in the HA [5].

\subsection{Disturbing influences: insertion of the viral fu- sion peptide into the target membrane bilayer}

The key role of the hydrophobic fusion peptide located at the N-terminal of HA2 in HA-mediated membrane merging has long been thought to involve an interaction with the target membrane that would induce destabilization at the contact zone. Indeed, several studies have shown that the peptide can penetrate target membranes prior to fusion, strongly suggesting that this might be the first step in membrane mixing per se (Fig. 1B) [11,23,24], although it should be mentioned that fusion peptide insertion alone may not lead to membrane mixing, and that these pre-fusion interactions are much less evident when biological membranes are used as targets for the virus [14].

One major difficulty in rationalizing fusion peptide insertion into the target bilayer was the fact that the peptide is located at the base of the HA at neutral $\mathrm{pH}$ (Fig. 1A, $\mathrm{A}^{\prime}$ ). However, predictions with synthetic peptides [25] and partial solving of the low $\mathrm{pH}$ structure of HA [26], have shown that drastic rearrangements take place, including major transitions in several regions of the molecule from loop to helical and vice versa. This results in the formation of a long helix that can project upward [25,26]. Since the fusion peptide is located at the tip of the low $\mathrm{pH}$-generated helix, it can thus be propelled into the target membrane (Fig. 1B). Peptide insertion, possibly in an oblique position [27,28], may also be made easier by the fact that fusion-active HA assumes a tilted position in the viral envelope at low pH (Fig. 1B,C) [29].

As discussed earlier, lack of a target membrane, inadequate positioning of the virus on the target membrane surface, or absence of HA binding to specific receptors result in viral inactivation at low $\mathrm{pH}$. It has been proposed that this process results from insertion of the fusion peptide into the viral envelope, after the low $\mathrm{pH}$-formed long HA2 helix changes direction [30,31]. Therefore, in the absence of a target membrane that could modulate specific HA binding, insertion of the fusion peptide into bilayers would take place in an random fashion (Fig. $\left.1 B^{\prime}, C^{\prime}\right)[15,16]$. In the presence of the target bilayer, the two postulated movements of the fusion peptide (projection towards the target, followed by reversal of orientation back to the envelope) might be the main driving force in membrane merging $[3,26,32]$. These movements could be made possible by a change from helix to loop in a region of the HA stem that could thus function as a 'hinge' (Fig. 1C-F).

It should be stressed that structural data are available for only a soluble fragment of HA2 at low $\mathrm{pH}$ [26], and still lacking is concrete knowledge of the way the fusogenic protein is placed in the viral envelope during fusion, and, more importantly, the exact structure of the fusion peptide when interacting with a target membrane. Using synthetic peptides corresponding to the N-termini of several HAs both $\alpha$-helixes and $\beta$-strands were detected, with a slight predominance of the former structure [28]. Moreover, it was also shown that lower fusion activity correlated with an increase in $\beta$-strands, suggesting that the peptide, which assumes a random conformation in aqueous solution, may form a helix when in contact with the target membrane [28]. Such a mechanism has been proposed to actually assist in bringing together the fusing membranes [33].

\subsection{Key cooperations: participation of several HA trimers at the fusion site}

Various lines of indirect evidence have implied that several HA trimers had to be involved in each fusion site (therefore suggesting the concerted action of several fusion peptides), and a lag phase, of varying duration, between acidification and membrane merging has therefore been interpreted as the required time for low $\mathrm{pH}$ association of trimers (Fig. 1B,C) $[17,34,35]$. Recent calculations based on kinetic analysis have yielded a minimal number of three [36] and six [37] trimers per fusion event. It should also be mentioned that alterations in fusion 
peptide interaction with target bilayers during the lag phase and/or in the very early stages of the fusion itself, possibly as the result of trimer organization at the contact site, have been proposed [11,14]. In the absence of specific HA binding, trimer organization, as well as fusion peptide penetration into membranes, would occur at random, thus explaining the low fusion activity of influenza virus towards membranes lacking sialic acid receptors $[6,15]$.

\subsection{A question of bulk: overcoming physical barriers to final contact between membranes}

Once HA higher order complexes are formed, the process of membrane merging per se can take place. However, a major hurdle to be overcome concerns the presence at the fusion site of several very bulky glycoproteins, that can act as steric barriers to membrane contact, an issue overlooked in most models because the HA is only partially represented (typically only the low $\mathrm{pH}$ fragment of known structure, attached to a transmembrane anchor, is depicted). There are two processes that may contribute to solve this problem. On one hand, the tilted position of HA has already been mentioned [29]. On the other hand, trimer head dissociation could again be important in removing the globular HA1 subunits from the fusion site (Fig. 1B,C,D). If the globular heads remain (as postulated in the model) attached to target membrane receptors, opening of the trimers could directly promote close contact between the apposing membranes. In this respect, it should be noted that even low $\mathrm{pH}$-inactivated virions retain most of their binding ability, at neutral and acidic $\mathrm{pH}$ [5]. Both processes mentioned above could explain the establishment of direct contact between the viral envelope and the endosome membrane.

2.6. Involvement of proteins and lipids: early intermediates in the coalescence of aqueous compartments

Although HA-induced fusion is protein-mediated, it had already been suggested that the membrane fusion event itself would take place in target membrane protein-free areas [17]. The use of mutant GPI-anchored HAs has shown that lack of a protein transmembrane domain induced hemifusion (i.e., mixing of only the outer monolayers of fusing membranes), therefore implying that a lipidic intermediate state was involved [38]. However, it was also possible that these observations resulted from the lack of the transmembrane region, and were not physiologically relevant.

Another favored model for the architecture of the fusion site postulated that initial contact between aqueous compartments would take place via a protein-lined 'fusion pore' $[37,39,40]$, akin to what has been modeled in the case of fusion during exocytosis [41]. These models arose mainly from the observation that aqueous contact between compartments could be established before exchange of lipids between the two membranes, a possible problem in the interpretation of the data being that pore opening assays were more sensitive than the equivalent lipid-mixing techniques. This would help explain the fact that lipids have been recently shown to be paramount in membrane merging, and especially that they can modulate the bilayer mixing process before the establishment of any aqueous contact between compartments defined by fusing membranes can be detected [42]. The observation that hemifusion induced by GPI-anchored HA can lead to complete fusion in the presence of modulators of membrane curvature seems to point in the same direction [43]. Thus, it seems likely that the initial contact between aqueous compartments will be mediated by lipids (possibly forming a highly curved hourglass-like 'stalk' intermediate), whose behavior has been directly or indirectly conditioned by the HA, namely by its fusion peptide (Fig. 1D). Following the formation of a hemifusion structure, this will likely evolve into a single monolayer contact region [43] that later collapses into a small fusion pore (Fig. 1E). Interestingly, the fusion peptide has also been implicated in pore widening [44], suggesting that its presence in the fusion site might be important in the final consummation of membrane fusion (Fig. 1E,F).

\section{Perspectives}

In the model for HA-mediated membrane fusion presented above, we have integrated information obtained from many studies, on a wide variety of 
experimental systems, in an effort to produce a detailed account of the process at the molecular level. Accordingly, we have taken into consideration all aspects of HA function, including those often overlooked by other authors for reasons of simplicity, such as the modulating role of target membrane sialic acid on fusion activity, and the role of globular head dissociation in the merging of bilayers. The picture is however not complete, and will likely be revised as more information on the precise structure of $\mathrm{HA}$ at the fusion site is unveiled, especially since low $\mathrm{pH}$ conformation structure is only known for fragments of the protein. This pictorial hypothesis could be useful in bringing together structural considerations and evidence obtained from kinetic studies.

As far as other biological fusion reactions are concerned, it is possible that HA mediated fusion could serve as a broader paradigm. However, any extrapolations should be made with caution, as it is becoming clear that, although most viral fusion proteins share many distinctive characteristics, the way they perform their respective membrane merging events may be quite different $[1,2,45]$.

Nevertheless, the sperm integral membrane protein fertilin has been described as a likely candidate for the fusion factor responsible for membrane merging during fertilization, since it has been shown to possess several of the characteristics common to many viral fusion proteins. These characteristics include synthesis as an inactive precursor, existence of tightly linked subunits, and possibility of formation of highorder oligomers, as well as existence of a putative fusion peptide in the $\alpha$ subunit of the protein (for recent reviews see Refs. [45,46]), although recent observations suggest that the human $\alpha$ fertilin might be non-functional [47], and that fusion during fertilization could involve other proteins [48].

But if events such as fertilization or myoblast fusion could be considered similar to cell infection by lipid-enveloped viruses, since both involve fusion of structures facing an extracellular medium, it is unclear whether the same type of rationalism could be imported to intracellular membrane fusion. For example, regulation of intracellular fusion events involved in membrane trafficking might be accomplished solely by elaborate mechanisms regarding fusion inhibition of naturally unstable membranes, until proper docking/priming can be achieved, thus precluding the need for specific fusion proteins. Nevertheless, the study of these intracellular events has been hindered, not only by the complexity of the system, which makes it difficult to isolate (and properly reconstitute) any given fusion event for kinetic analysis, but also by the lack of precise structural data on the many proteins involved. However, it has been proposed that the synaptic vesicle membrane protein synaptobrevin may mediate membrane fusion during the exocytotic release of neurotransmitters (in all likelihood, the best characterized intracellular fusion event), possibly via a fusion peptide akin to those found in viral proteins [for a recent review, see Ref. [49]]. Although more information is still needed, preliminary results suggest that synaptobrevin could indeed be directly involved in membrane mixing at the synapse [50].

Also of importance are the recent findings concerning the active participation of lipids in HA-mediated membrane fusion. Such findings are remarkable in that lipids have usually been considered secondary players in a protein-regulated process, their presence only being rationalized in terms of the maintenance of stable platforms (membranes) and/or compartments suitable for protein function. However, recent studies have demonstrated that several biological membrane fusion events (including exocytosis and viral fusion) can be directly influenced by membrane lipids [42,43,51]. Concomitantly, it is becoming increasingly clear that intracellular membrane trafficking may also involve changes in lipid bilayers that could facilitate vesicle budding and/or fusion. In this respect, activation of phospholipase D during intracellular signaling, with subsequent production of phosphatidic acid (a well known fusogenic lipid), is the best example of possible lipid participation in membrane destabilization during trafficking [52]. It is also likely that this previously not sufficiently appreciated area of research will yield many new developments in years to come.

\section{Acknowledgements}

Work in our laboratory was supported by JNICT (Portugal) and by a NATO Collaborative Research Grant, CRG 900333. 


\section{References}

[1] D. Hoekstra, M.C.P. Lima, Membrane interactions of HIV: Implications for pathogenesis and therapy in AIDS, in: R.C. Aloia, C.C. Curtain, (Eds.), Advances in Membrane Fluidity, Vol. 6, Wiley-Liss, New York, 1992, pp. 71-97.

[2] Y. Gaudin, R.W.H. Ruigrok, J. Brunner, J. Gen. Virol. 76 (1995) 1541-1556.

[3] J. White, Cold Spring Harbor Symp. Quant. Biol. 50 (1995) 581-588.

[4] M.C.P. Lima, J. Ramalho-Santos, M.F. Martins, A.P. Carvalho, V. Bairos, S. Nir, Eur. J. Biochem. 205 (1992) 181-186.

[5] J. Ramalho-Santos, S. Nir, N. Düzgünes, A.P. Carvalho, M.C.P. Lima, Biochemistry 32 (1993) 2771-2779.

[6] J. Ramalho-Santos, M.C.P. Lima, S. Nir, J. Biol. Chem. 271 (1996) 23902-23906.

[7] D.C. Wiley, J.J. Skehel, Annu. Rev. Biochem. 56 (1987) 675-697.

[8] J.M. White, I.A. Wilson, J. Cell. Biol. 105 (1987) 28872896.

[9] M.-J. Gething, R.W. Doms, D. York, J. White, J. Cell Biol. 102 (1986) 11-23.

[10] S.A. Tatulian, L.K. Tamm, J. Mol. Biol. 260 (1996) $312-$ 316.

[11] M. Tsurudome, R. Glück, R. Graf, R. Falchetto, U. Schaller, J. Brunner, J. Biol. Chem. 267 (1992) 20225-20232.

[12] A. Puri, F.P. Booy, R.W. Doms, J.M. White, R. Blumenthal, J. Virol. 64 (1990) 3824-3832.

[13] C.C. Pak, M. Krumbiegel, R. Blumenthal, J. Gen. Virol. 75 (1994) 395-399.

[14] C.C. Pak, M. Krumbiegel, R. Blumenthal, Y. Raviv, J. Biol. Chem. 269 (1994) 14614-14619.

[15] M.C.P. Lima, J. Ramalho-Santos, D. Flasher, V.A. Slepushkin, S. Nir, N. Düzgünes, Biochim. Biophys. Acta 1236 (1995) 323-330.

[16] T. Stegmann, I. Bartoldus, J. Zumbrunn, Biochemistry 34 (1995) 1825-1832.

[17] H. Ellens, J. Bentz, D. Mason, F. Zhang, J. White, Biochemistry 29 (1990) 9697-9707.

[18] D. Alford, H. Ellens, J. Bentz, Biochemistry 33 (1994) 1977-1987.

[19] T. Stegmann, J. White, A. Helenius, EMBO J. 9 (1990) 4231-4241.

[20] T. Stegmann, Curr. Biol. 4 (1994) 551-554.

[21] L. Godley, J. Pfeiffer, D. Steinhauer, B. Ely, G. Shaw, R. Kaufmann, E. Suchanek, C. Pabo, J.J. Skehel, D.C. Wiley, Cell 68 (1992) 635-645.

[22] G.W. Kemble, D.L. Bodian, J. Rosé, I.A. Wilson, J.M. White, J. Virol. 66 (1992) 4940-4950.

[23] C. Harter, P. James, T. Bächi, G. Semenza, J. Brunner, J. Biol. Chem. 264 (1989) 6459-6464.

[24] T. Stegmann, J.M. Delfino, F.M. Richards, A. Helenius, J. Biol. Chem. 266 (1991) 18404-18410.
[25] C.M. Carr, P.S. Kim, Cell 73 (1993) 823-832.

[26] P.A. Bullough, F.M. Hughson, J.J. Skehel, D.C. Wiley, Nature 371 (1994) 37-43.

[27] J. Lüneberg, I. Martin, F. Nüßler, J.-M. Ruysschaert, A. Herrmann, J. Biol. Chem. 270 (1995) 27606-27614.

[28] C. Gray, S.A. Tatulian, S.A. Wharton, L.K. Tamm, Biophys. J. 70 (1996) 2275-2286.

[29] S.A. Tatulian, P. Hinterdorfer, G. Baber, L.K. Tamm, EMBO J. 14 (1995) 5514-5523.

[30] T. Weber, G. Paesold, C. Galli, R. Mischler, G. Semenza, J. Brunner, J. Biol. Chem. 269 (1994) 18353-18358.

[31] S.A. Wharton, L.J. Calder, R.W.H. Ruigrok, J.J. Skehel, D.A. Steinhauer, D.C. Wiley, EMBO J. 14 (1995) 240-246.

[32] F.M. Hughson, Curr. Biol. 5 (1995) 265-274.

[33] J. Zimmerberg, S.S. Vogel, L.V. Chernomordik, Annu. Rev. Biophys. Biomol. Struct. 22 (1993) 433-466.

[34] S.J. Morris, D. Sarkar, J.M. White, R. Blumenthal, J. Biol. Chem. 264 (1989) 3972-3978.

[35] M.J. Clague, C. Schoch, R. Blumenthal, J. Virol. 65 (1993) 2402-2407.

[36] T. Danieli, S. Pelletier, Y.I. Henis, J.M. White, J. Cell Biol. 133 (1996) 559-569.

[37] R. Blumenthal, D. Sarkar, S. Durell, D.E. Howard, S.J. Morris, J. Cell Biol. 135 (1996) 63-71.

[38] G.W. Kemble, T. Danieli, J. White, Cell 76 (1994) 383-391.

[39] F.W. Tse, A. Iwata, W. Almers, J. Cell Biol. 121 (1993) 543-552.

[40] J. Zimmerberg, R. Blumenthal, D.P. Sarkar, M. Curran, S.J. Morris, J. Cell Biol. 127 (1994) 1885-1894.

[41] J.R. Monck, J.M. Fernandez, J. Cell Biol. 119 (1992) 13951404.

[42] L.V. Chernomordik, E.A. Leikina, V. Frolov, P. Bronk, J. Zimmerberg, J. Cell Biol. 136 (1997) 781-793.

[43] G.B. Melykian, S.A. Brener, D.C. Ok, F.S. Cohen, J. Cell Biol. 136 (1997) 995-1005.

[44] C. Schoch, R. Blumenthal, J. Biol. Chem. 268 (1993) 9267-9274.

[45] L.D. Hernandez, L.R. Hoffman, T.G. Wolfsberg, J.M. White, Annu. Rev. Cell Dev. Biol. 12 (1996) 627-661.

[46] W.J. Snell, J.M. White, Cell 85 (1996) 629-637.

[47] J.A. Jury, J. Frayne, L. Hall, Biochem J. 321 (1997) 577581.

[48] D.G. Myles, P. Primakoff, Biol. Rep. 56 (1997) 320-327.

[49] N. Calakos, R.H. Scheller, Physiol. Rev. 76 (1996) 1-29.

[50] M.T. Almeida, J. Ramalho-Santos, C.R. Oliveira, M.C.P. Lima, Biochem. Biophys. Res. Commun. 236 (1997) 184188.

[51] L.V. Chernomordik, S.S. Vogel, A. Sokoloff, H.O. Onaran, E.A. Leikina, J. Zimmerberg, FEBS Lett. 318 (1993) 71-76.

[52] M. Liscovich, J. Lipid Mediators Cell Signalling 14 (1996) 215-221. 